\title{
Transient x-ray absorption spectroscopy of hydrated halogen atom
}

\author{
Christopher G. Elles, ${ }^{\text {a) }}$ llya A. Shkrob, ${ }^{\text {b) }}$ and Robert A. Crowell ${ }^{\text {() }}$ \\ Chemistry Division, Argonne National Laboratory, Argonne, Illinois 60439, USA \\ Dohn A. Arms and Eric C. Landahl \\ Advanced Photon Source, Argonne National Laboratory, Argonne, Illinois 60439, USA
}

(Received 20 September 2007; accepted 30 November 2007; published online 11 February 2008)

\begin{abstract}
Time-resolved x-ray absorption spectroscopy has been used to observe the transient species generated by one-photon detachment of an electron from aqueous bromide. The $K$-edge spectrum of the short-lived $\mathrm{Br}^{0}$ atom exhibits a resonant $1 s-4 p$ transition that is absent for the $\mathrm{Br}^{-}$precursor. The strong $1 s-4 p$ resonance suggests that there is very little charge transfer from the solvent to the open-shell atom, whereas weak oscillations above the absorption edge indicate that the solvent shell around a neutral $\mathrm{Br}^{0}$ atom is defined primarily by hydrophobic interactions. These conclusions are in agreement with Monte Carlo and quantum chemical simulations of the solvent structure. (C) 2008 American Institute of Physics. [DOI: 10.1063/1.2827456]
\end{abstract}

Reactions of small inorganic radicals in aqueous solution are important for aerosol, marine, photo- and radiation chemistry. The reactivity of such radicals depends on their interaction with polar water molecules. As there are a limited number of experimental methods for studying the hydration of short-lived radicals lacking a strong chromophore, new techniques capable of yielding direct structural information with atomic resolution are required. Time-resolved x-ray scattering $^{1}$ and $\mathrm{x}$-ray absorption spectroscopy ${ }^{2-7}$ (XAS) are such techniques. In this Communication, we report the study of the hydration of a simple open-shell system, the $\mathrm{Br}^{0}$ atom, using transient XAS.

Details about the hydration of halogen atoms are not well known. ${ }^{2,3}$ The hydration of halogen atoms $\left(X^{0}\right)$ is very different from the hydration of negatively charged halide anions $\left(X^{-}\right)$. Whereas the anion forms strong hydrogen bonds with several water molecules in the first solvent shell [Fig. 1(a)], hydrophobic effects dominate the solvation of neutral halogen atoms [Fig. 1(b)]. The bromine atom resides in a solvent cavity and interacts with the solvent to give a charge transfer (CT) absorption band at $4.6 \mathrm{eV}{ }^{8}$ The CT absorption promotes an electron from the water molecule(s) onto the halogen atom [Fig. 1(c)]. In the case of $\mathrm{Cl}^{0}$, electron spin resonance measurements ${ }^{9}$ suggest that the $\mathrm{Cl}^{0}$ atom and one of the water molecules form a two center, three electron $\left(\sigma^{2} \sigma^{* 1}\right)$ bond in the ground state. Similar bonding may also occur for $\mathrm{Br}^{0}$ and $\mathrm{I}^{0}$ in water. ${ }^{10}$ The much stronger halogenhalogen bonding between a halogen atom and its anion is also a two center, three electron bond [Fig. 1(d)].

Recently, Pham et al. ${ }^{7}$ reported XAS measurements of $\mathrm{I}^{0}$ atoms following biphotonic electron detachment from aqueous iodide. They observe a prominent change of the spectra at the $L_{1}$ and $L_{3}$ absorption edges, but their interpretation is

\footnotetext{
${ }^{\text {a) }}$ Present address: Department of Chemistry, University of Southern California, Los Angeles, CA 90089. Electronic mail: elles@usc.edu.

${ }^{b)}$ Electronic mail: shkrob@anl.gov.

${ }^{c}$ Present address: Chemistry Bldg. 555, Brookhaven National Laboratory, Upton, NY 11973-5000. Electronic mail: crowell@bnl.gov.
}

complicated by the reaction of $\mathrm{I}^{0}$ with excess $\mathrm{I}^{-}$to form a substantial amount of $\mathrm{I}_{2}{ }^{-}$and $\mathrm{I}_{3}{ }^{-}$within the $\sim 80 \mathrm{ps}$ duration of the x-ray pulse. Rapid formation of molecular anions in their experiment is a consequence of the high concentration of $\mathrm{I}^{-}\left(500 \mathrm{~mol} / \mathrm{m}^{3}\right)$, which is necessary to overcome inefficient two-photon absorption. In contrast, efficient onephoton electron detachment from $\mathrm{Br}^{-}$at $200 \mathrm{~nm}$ (Ref. 11) and strong absorption at the $K$ edge of bromine allow us to use dilute aqueous solutions in which the reaction of $\mathrm{Br}^{0}$ with excess anions is much slower.

The present experiment uses the laser pump-X-ray probe capabilities of beamline 7ID of the Advanced Photon Source at Argonne. Fourth harmonic generation of the output from an amplified Ti:sapphire laser provides $5 \mu \mathrm{J}$ pulses of $200 \mathrm{~nm}$ light with a repetition rate of $1 \mathrm{kHz}$. A $\mathrm{MgF}_{2}$ lens focuses the ultraviolet light to a diameter of $95 \mu \mathrm{m}$ at the sample, where it crosses the x-ray beam at an angle of $4^{\circ}$. The laser is synchronized with the synchrotron by an active feedback control loop that adjusts the laser oscillator cavity length. The relative delay between laser and x-ray pulses is controlled electronically. The synchrotron provides tunable $\mathrm{X}$-ray pulses with a duration of $\sim 80 \mathrm{ps}$ and a repetition rate of $6.54 \mathrm{MHz}$ ( 24 bunch mode). $\mathrm{X}$ rays from the undulator pass through a tunable diamond monochromator $(\Delta E / E=5$ $\times 10^{-5}$ ) before a Kirkpatrick-Baez mirror pair focuses them to a spot size of about $25 \mu \mathrm{m}$ in the sample. The sample is a $100 \mu \mathrm{m}$ thick liquid jet of $5-10 \mathrm{~mol} / \mathrm{m}^{3} \mathrm{NaBr}$ solution with the flat surface of the jet rotated $45^{\circ}$ relative to the $\mathrm{x}$-ray beam. Se filters absorb elastically scattered $\mathrm{x}$ rays and $\mathrm{a}$ gated avalanche photodiode detector on each side of the jet monitors the bromine $K_{\alpha}$ fluorescence. We record fluorescence count rates for the two x-ray pulses immediately following the laser pulse $(\Delta t, \Delta t+153 \mathrm{~ns})$ and normalize the signal to account for variations of the x-ray flux. Scanning the incident energy gives the spectra at each delay time.

Figure 2(a) compares the static spectrum of the bromide solution with the transient spectrum at 1 ns delay. The conversion of a fraction of $\mathrm{Br}^{-}$anions to neutral $\mathrm{Br}^{0}$ atoms by 
<smiles>CC(C)(C)C(O)OC(O)C(O)C(O)O</smiles>

(b)<smiles>OC1OCOCOC1O</smiles>

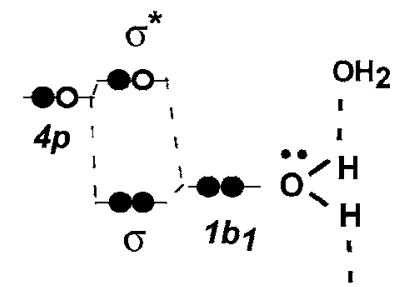

(c) $\mathrm{Br}^{\mathrm{O}}$

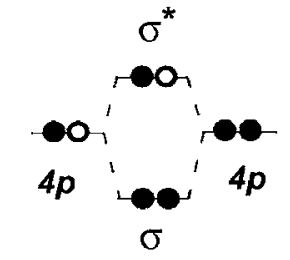

(d) $\mathrm{Br}^{\mathbf{O}}$

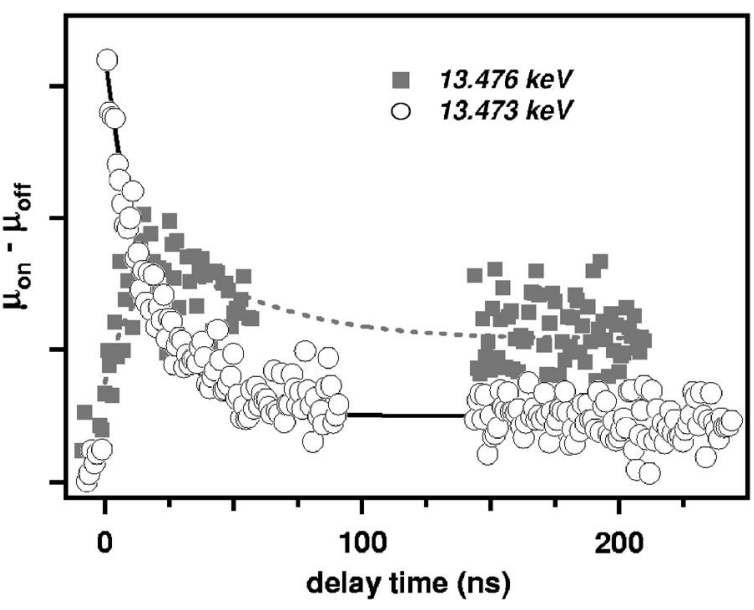

FIG. 3. Transient $\mathrm{x}$-ray absorption difference signals $\mu_{\mathrm{on}}-\mu_{\text {off }}$ for $4.9 \mathrm{~mol} / \mathrm{m}^{3}$ solution of $\mathrm{NaBr}$ observed at $13.473 \mathrm{keV}$ (open circles) and $13.476 \mathrm{keV}$ (filled squares). The lines are to guide the eye.

FIG. 1. Sketches of the solvent structure around (a) $\mathrm{Br}^{-}$and (b) $\mathrm{Br}^{0}$. Panels (c) and (d) show the schematic orbital diagrams for the $\mathrm{Br}^{0} \cdots \mathrm{OH}_{2}$ complex and $\mathrm{Br}_{2}^{-}$, respectively.

the $200 \mathrm{~nm}$ laser pulse is evident from the resonant $1 s-4 p$ transition below the bromine $K$ edge. Subtracting the static spectrum of $\mathrm{Br}^{-}$from the transient spectra at delays of 1 and 154 ns gives the difference spectra $\mu_{\text {on }}-\mu_{\text {off }}$ in Fig. 2(b). The spectra at the two delay times are different because nearly half of the $\mathrm{Br}^{0}$ atoms at $1 \mathrm{~ns}$ react with excess $\mathrm{Br}^{-}$to form $\mathrm{Br}_{2}^{-}$, while the other $\mathrm{Br}^{0}$ atoms recombine with the hydrated electron. Other than $\mathrm{Br}^{-}$, the predominant species at 1 and 154 ns are $\mathrm{Br}^{0}$ and $\mathrm{Br}_{2}{ }^{-}$, respectively. ${ }^{12}$

The difference spectra in Fig. 2(b) show that the resonant transition in $\mathrm{Br}_{2}^{-}$is $1.6 \mathrm{eV}$ higher in energy than the

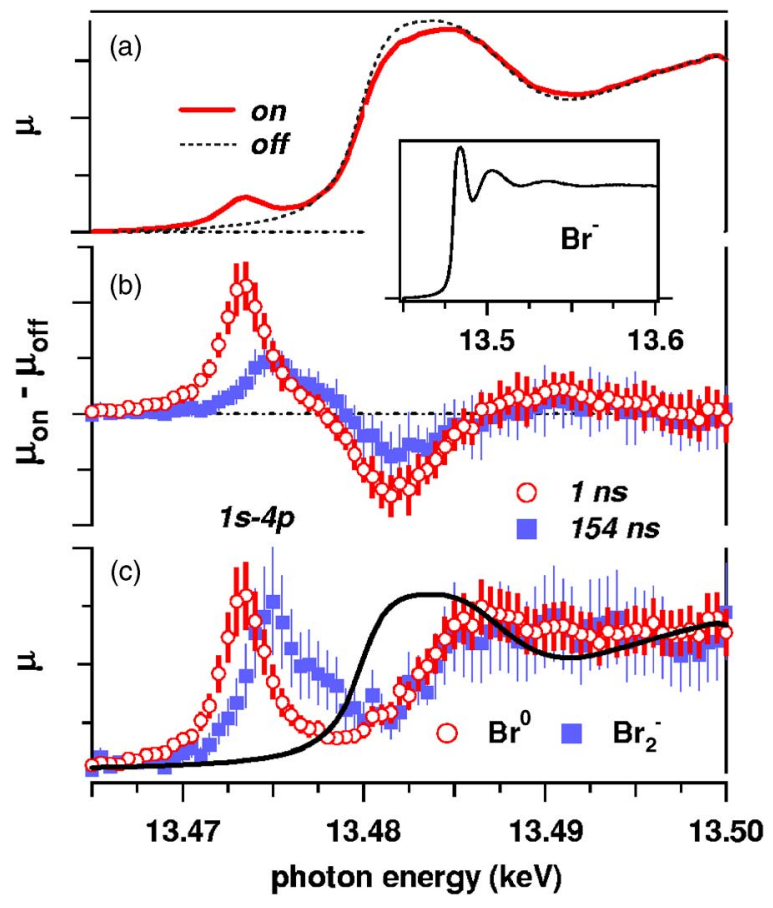

FIG. 2. (Color online) (a) Laser-on and laser-off $x$-ray absorption spectra of aqueous $\mathrm{Br}^{-}$at $\Delta t=1 \mathrm{~ns}$. The inset shows the static (laser-off) spectrum of $\mathrm{Br}^{-}$over a wider energy range. (b) The difference spectra $\mu_{\mathrm{on}}-\mu_{\text {off }}$ at delay times of $1 \mathrm{~ns}$ (open circles) and $154 \mathrm{~ns}$ (filled squares). (c) Reconstructed spectra of $\mathrm{Br}^{0}$ (open circles) and $\mathrm{Br}_{2}^{-}$(filled squares). The solid line is the absorption spectrum of $\mathrm{Br}^{-}$. Vertical bars indicate one standard deviation.

transition in $\mathrm{Br}^{0}$. Excitation of $\mathrm{Br}^{0}$ promotes a $1 s$ electron into the $4 p$ vacancy produced by detaching an electron from $\mathrm{Br}^{-}$, whereas the resonant transition for the $\mathrm{Br}_{2}^{-}$anion excites an electron to a $\sigma^{*}$ antibonding orbital [Figs. 1(c) and $1(\mathrm{~d})] .{ }^{13}$ The spectral shift of the resonant x-ray absorption energy allows us to observe the reaction kinetics of the transient $\mathrm{Br}^{0}$ atom. Figure 3 shows the time-dependent change in absorption at 13.473 and $13.476 \mathrm{keV}$. Absorption at the lower energy is predominantly due to $\mathrm{Br}^{0}$ atoms; therefore, the decay of the $\mathrm{x}$-ray absorbance indicates the loss of $\mathrm{Br}^{0}$ atoms as they recombine with hydrated electrons and react with $\mathrm{Br}^{-}$. Both $\mathrm{Br}^{0}$ and $\mathrm{Br}_{2}{ }^{-}$contribute to the absorption at $13.476 \mathrm{keV}$, where the initial increase of the signal is due to production of $\mathrm{Br}_{2}^{-}$and the slower partial decay is from the $\mathrm{Br}^{0}$ contribution. Importantly, these kinetics give an accurate estimate of the product concentrations and thus allow us to reconstruct the spectra of the transient species by subtracting the contribution from $\mathrm{Br}^{-}$. Details of the kinetic scheme and calculation of the conversion yields are given as supplemental material. ${ }^{12}$ The reconstructed spectra of $\mathrm{Br}^{0}$ and $\mathrm{Br}_{2}{ }^{-}$are shown in Fig. 2(c), along with the static spectrum of $\mathrm{Br}^{-}$. The $K$-edge absorption energy is $\sim 5 \mathrm{eV}$ higher for the transient species than for bromide. The higher energy for $\mathrm{Br}^{0}$ reflects the electrostatic attraction of the outgoing electron to the positively charged core.

Perhaps the most intriguing feature of the recovered $\mathrm{x}$-ray absorption spectrum of $\mathrm{Br}^{0}$ is the significantly shallower modulation above the $K$ edge relative to the $\mathrm{Br}^{-}$spectrum. A Monte Carlo (MC) simulation of the solvent structure provides helpful insight to understand this difference in the x-ray absorption fine structure (XAFS). The simulation includes 200 simple point charge/flex water molecules ${ }^{14}$ and a single $\mathrm{Br}^{0}$ or $\mathrm{Br}^{-}$in a supercell, with solute-water interaction potentials from Refs. 15 and 10, respectively. An ensemble of 1500 snapshots at $298 \mathrm{~K}$ were taken from 3 $\times 10^{7} \mathrm{MC}$ steps to give the $\mathrm{Br}-\mathrm{O}$ radial distribution functions (RDFs), $g_{\mathrm{Br}-\mathrm{O}}(r)$, in Fig. 4(a). (The proton contribution to the XAFS spectrum is negligible.) For $\mathrm{Br}^{-}$, the narrow peak at $3.2 \AA$ is due to strong hydrogen bonding $\left(\mathrm{Br}^{-} \cdots \mathrm{H}-\mathrm{OH}\right)$ between the anion and approximately six 

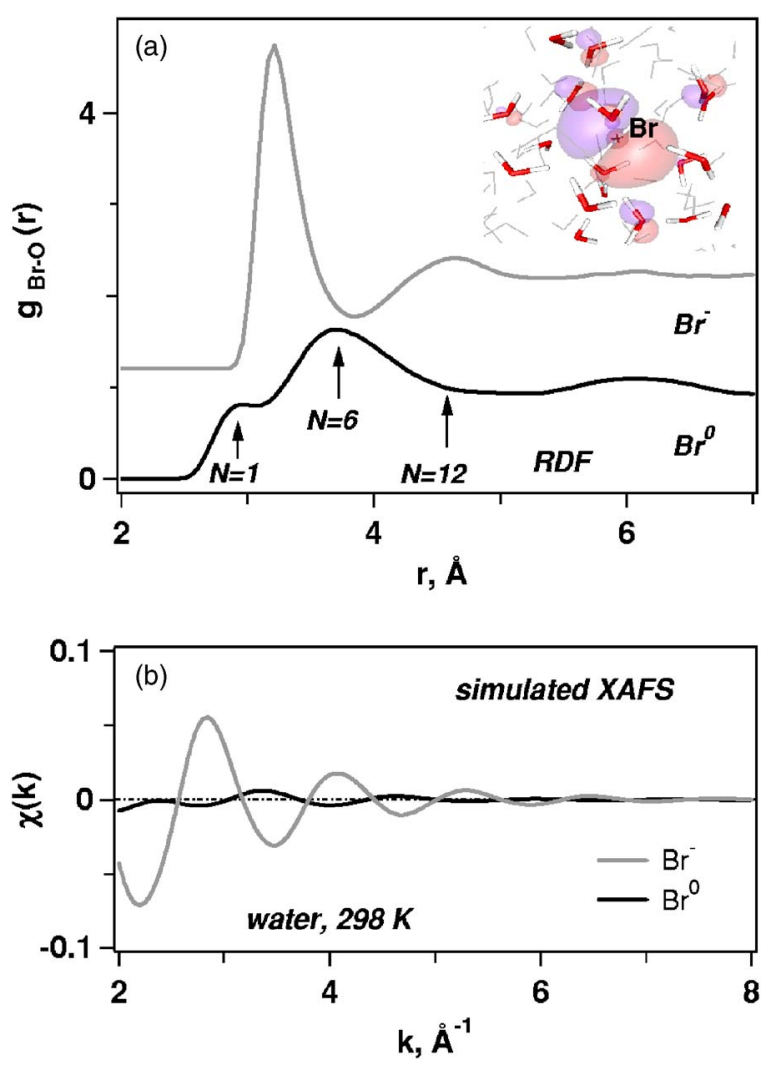

FIG. 4. (Color online) (a) Calculated $\mathrm{Br}-\mathrm{O}$ RDF for hydrated $\mathrm{Br}^{-}$and $\mathrm{Br}^{0}$. Oxygen coordination numbers $N$ are indicated by arrows. The inset shows the singly occupied orbital of the bromine atom inside the embedded water cluster from our $a b$ initio calculations. (b) The simulated XAFS spectra for hydrated $\mathrm{Br}^{-}$and $\mathrm{Br}^{0}$. The first oscillation corresponds to the energy range of Fig. 2(c).

water molecules in the first solvent shell. ${ }^{15,16}$ The RDF for the $\mathrm{Br}^{0}$ atom lacks this feature because the hydrophobic atom interacts weakly with the solvent. Instead, the atom occupies a nearly spherical cavity formed by $10-12$ water molecules that are hydrogen bonded to other water molecules in the first and second solvent shells. The only distinctive feature in this RDF is a shoulder at $3 \AA$ that corresponds to a weak $\mathrm{Br}^{0} \cdots \mathrm{OH}_{2}$ adduct involving a single water molecule. For other water molecules, the $\mathrm{Br}^{0}-\mathrm{O}$ distances are significantly longer, $\sim 3.7 \AA$.

Taking nuclear configurations from the $\mathrm{MC}$ ensemble for water molecules with $r(\mathrm{Br}-\mathrm{O})<8 \AA$, we simulate the XAFS spectra of $\mathrm{Br}^{-}$and $\mathrm{Br}^{0}$ using the program FEFF8 [Fig. 4(b)]. ${ }^{17}$ The highly organized hydrogen bonding structure of hydrated $\mathrm{Br}^{-}$gives deep oscillations in the static spectrum, but the magnitude of the oscillations is approximately ten times smaller for hydrated $\mathrm{Br}^{0}$ atoms. Although the calculation pertains to higher $\mathrm{x}$-ray energies than the present experiment covers (only the first oscillation is observed experimentally), the modulation is clearly much weaker in the reconstructed spectrum of $\mathrm{Br}^{0}$ than in the $\mathrm{Br}^{-}$spectrum [Fig. 2(c)]. The contribution of the $\mathrm{Br}^{0} \cdots \mathrm{OH}_{2}$ adduct to the XAFS spectrum is small compared with the contributions from the other 10-12 oxygen atoms in the first solvent shell.

Pham et al..$^{7}$ suggested that significant CT from a water molecule suppresses the $2 s-5 p$ resonance in the $L_{1}$ spectrum of $\mathrm{I}^{0}$ by as much as $70 \%$ relative to the absorption strength above the edge. No such dramatic suppression of the $1 s-4 p$ resonance is evident from our $K$-edge spectrum of $\mathrm{Br}^{0}$. Although the relative intensity of the preedge absorption is sensitive to the ratio of $\mathrm{Br}^{0} / \mathrm{Br}^{-}$that we use to reconstruct the spectrum, the actual $\mathrm{Br}^{0}$ yield would have to be a factor of 2 higher than we assume in order to give a $20 \%$ suppression below the absorption edge. This is unlikely given the close agreement of our calculated photoconversion yield (13\%) and the value obtained by fitting the decay of the $\mathrm{Br}^{0}$ signal in Fig. 3 (12\%-15\%).

To estimate the degree of $\mathrm{CT}$ in the $\mathrm{Br}^{0}$-water adduct, water clusters that include all solvent molecules with $r\left(\mathrm{Br}^{0}-\mathrm{O}\right)<5.5 \AA$ were extracted from the MC simulation. Water molecules outside of the extracted cluster were replaced by fractional point charges and Hartee-Fock calculations of the "embedded" clusters using a $6-311++\mathrm{G}^{* *}$ basis set give a Mulliken charge of $-(0.07-0.08)$ on the $\mathrm{Br}^{0}$ atom, which would suppress the preedge feature very little. The same calculation indicates that $92 \%-95 \%$ of the unpaired electron density resides on the bromine atom. Configuration interaction with single excitations calculations for the embedded clusters indicate that weak $\mathrm{CT}$ is sufficient to account for the observed absorption band in the ultraviolet ${ }^{8}$ (with a calculated oscillator strength of 0.2 and transition energy of $5.6 \mathrm{eV})$.

In conclusion, we report the transient $\mathrm{x}$-ray absorption spectrum of the short-lived hydrated $\mathrm{Br}^{0}$ atom and follow its reaction to form $\mathrm{Br}_{2}{ }^{-}$. The solvent shell around $\mathrm{Br}^{0}$ is defined primarily by weak hydrophobic interactions, with the atom residing within a large "bubble." Our simulations suggest that a single solvent molecule directly interacts with the atom to form a weak $\sigma^{2} \sigma^{* 1}$ bond. Although charge transfer in the ground state of the $\mathrm{Br}^{0}$-water adduct is too weak to noticeably suppress the $1 s-4 p$ resonance in the $\mathrm{x}$-ray absorption spectrum, the previously observed ${ }^{8} \mathrm{CT}$ band for neutral bromine atoms in water supports the formation of an adduct. The XAFS signature of the weak $\mathrm{Br}^{0}$-water complex is obscured by scattering from 10-12 unbound water molecules in the hydration shell. This study paves the way to observation and characterization of the solvent structure for other shortlived radical intermediates by means of time-resolved XAS.

We thank S. Ross for help with avalanche photodiode detectors, E. Dufresne and D. Walko for beamline assistance, L. Young and her colleagues for use of their instruments, and P. Jungwirth, C. Bressler, R. Saykally, P. D'Angelo, and S. Bradforth for helpful discussions. Use of the Advanced Photon Source was supported by the U.S. Department of Energy, Office of Basic Energy Sciences, under Contract No. DEAC02-06CH11357.

\footnotetext{
${ }^{1}$ A. Plech et al., Phys. Rev. Lett. 92, 125505 (2004).

${ }^{2}$ C. Bressler et al., J. Chem. Phys. 116, 2955 (2002).

${ }^{3}$ C. Bressler and M. Chergui, Chem. Rev. (Washington, D.C.) 104, 1781 (2004).

${ }^{4}$ L. X. Chen, Angew. Chem., Int. Ed. 43, 2886 (2004).

${ }^{5}$ W. Gawelda et al., Phys. Rev. Lett. 98, 057401 (2007).

${ }^{6}$ T. Lee et al., J. Chem. Phys. 122, 1 (2005).

${ }^{7}$ V.-T. Pham et al., J. Am. Chem. Soc. 129, 1530 (2007).

${ }^{8}$ A. Treinin and E. Hayon, J. Am. Chem. Soc. 97, 1716 (1975).
} 
${ }^{9}$ M. D. Sevilla et al., J. Phys. Chem. A 101, 2910 (1997).

${ }^{10}$ M. Roeselova, U. Kandor, and P. Jungwirth, J. Phys. Chem. A 104, 6523 (2000).

${ }^{11}$ R. Lian et al., J. Phys. Chem. A 110, 9071 (2006).

${ }^{12}$ See EPAPS Document No. E-JCPSA6-128-024802 for supplementary information. This document can be reached through a direct link in the online article's HTML reference section or via the EPAPS homepage (http://www.aip.org/pubservs/epaps.html).

${ }^{13}$ D. Zehavi and J. Rabani, J. Phys. Chem. 76, 312 (1972).

${ }^{14}$ Y. Wu, H. L. Tepper, and G. A. Voth, J. Chem. Phys. 124, 024503 (2006).

${ }^{15}$ P. D'Angelo et al., J. Chem. Phys. 100, 985 (1994).

${ }^{16}$ P. J. Merkling et al., J. Chem. Phys. 119, 6647 (2003).

${ }^{17}$ A. L. Ankudinov et al., Phys. Rev. B 58, 7565 (1998). 